\title{
Strong $\lambda$-lemma and second species periodic solutions of the 3-body problem
}

\section{Piero Negrini}

Dipartimento Matematico "G. Castelnuovo", La Sapienza, Universitá di Roma

E-mail address: negrini@mat.uniroma1.it

\begin{abstract}
Quite recently Bolotin and N. developed a variational approach to the existence of second species periodic solutions for the nonrestricted 3 body problem [2]. An important part of this paper is based on an analog of Shilnikov Lemma (also called strong $\lambda$ - lemma) for hamiltonian systems possessing a non degenerate normally hyperbolic critical symplectic manifold. The complete proof of the Lemma was postponed to a future publication [3]: in this paper we anticipate a part of the results.
\end{abstract}

\section{Introduction}

Consider the plane 3 -body problem with masses $m_{1}, m_{2}, m_{3}, m_{3}$ much larger than $m_{1}, m_{2}$, i.e. $\mu=\left(m_{1}+m_{2}\right) / m_{3}$ is a small parameter. Without loss of generality set

$$
m_{3}=1, \quad m_{1}=\mu \alpha_{1}, \quad m_{2}=\mu \alpha_{2}, \quad \alpha_{1}+\alpha_{2}=1 .
$$

Let $r_{i} \in \mathbb{R}^{2}$ be positions of the bodies $m_{i}$ and assume that the center of mass is at the origin. Call

$$
q_{1}=r_{1}-r_{3}, q_{2}=r_{2}-r_{3}
$$

the positions of $m_{1}, m_{2}$ with respect to $m_{3}$ (Poincaré's heliocentric coordinates) and let $p_{i}=\alpha_{i} \dot{r}_{i}$ be their scaled momenta. The motion of $m_{1}, m_{2}$ with respect to $m_{3}$ is described by hamiltonian system $\left(H_{\mu}\right)$ :

$$
H_{\mu}(q, p)=H_{0}(q, p)+\mu h(q, p),
$$


where

$$
\begin{aligned}
H_{0} & =\frac{\left|p_{1}\right|^{2}}{2 \alpha_{1}}-\frac{\alpha_{1}}{\left|q_{1}\right|}+\frac{\left|p_{2}\right|^{2}}{2 \alpha_{2}}-\frac{\alpha_{2}}{\left|q_{2}\right|} \\
h & =\frac{\left|p_{1}+p_{2}\right|^{2}}{2}-\frac{\alpha_{1} \alpha_{2}}{\left|q_{1}-q_{2}\right|} .
\end{aligned}
$$

In (1.1) (1.2), $q=\left(q_{1}, q_{2}\right), p=\left(p_{1}, p_{2}\right), p_{i} \in \mathbb{R}^{2}, q_{i} \in Q_{i}=\mathbb{R}^{2} \backslash\{0\}$. The configuration space of the perturbed hamiltonian system $\left(H_{\mu}\right)$ is $Q \backslash \Delta$, where

$$
Q=Q_{1} \times Q_{2}, \Delta=\left\{q \in Q: q_{1}=q_{2}\right\} .
$$

so that $\Delta$ represents collisions of $m_{1}, m_{2}$. Therefore $\mu h(q, p)$ is the perturbation of the unperturbed hamiltonian system $\left(H_{0}\right)$ which describes 2 uncoupled Kepler problems.

Close to the collisional set the classical perturbation theory does not work: $h$ becomes large, and so it can not be ignored even in the first approximation. However the neighborhood of $\Delta$ is very important to build up special periodic solutions, the so called second species periodic solutions. The idea of exploiting the singularity in $H_{\mu}$ belongs to Poincaré who only sketched the proof of their existence.

For small $\mu>0$ but small, the orbits of the 3 body problem can be described as follows. The bodies $m_{1}, m_{2}$ move close to nearly Kepler orbits and almost collide. After the near collision, they start moving nearly along a new pair of Kepler ellipses passing through the collision point. If the new frequencies are still incommensurable, then new Kepler orbits will again nearly collide, and the process starts again. Considering a periodic chain of near collisions we can gain a second species periodic solution. Rigorous proofs of the existence of such solutions appeared much later and only for the restricted 3 body problem (see for instance [1], [4], [5]) until the existence of second species periodic solutions for the nonrestricted 3 body problem has been studied in [2]. To investigate the orbits close to the collisional set the authors made use of a Lemma which is a generalization of Shilnikov Lemma [7] to systems with a normally hyperbolic critical manifold. In the next Section we present the complete proof of the Lemma (see Theorem (2.1)) and in the last Section we show its application to the 3 body problem.

\section{The Shilnikov Lemma}

We begin the Section recalling some results of the hyperbolic theory for systems possessing a non degenerate normally hyperbolic critical manifold (see $[2])$.

Let $n_{0}, n_{-}, n_{+}$be three positive integers, let $r$ be a positive number, $r \in$ $(0,1)$. Let $\mathcal{D}$ be an open subset of $\mathbb{R}^{n_{0}}$ having compact closure and for any

São Paulo J.Math.Sci. 6, 2 (2012), 351-363 
$z \in \mathcal{D}$ let $B_{2 r}^{0}(z)$ in $R^{n_{0}}$ be the ball of center $z$, radius $2 r$. Let $\mathcal{M}$ be a compact set such that $\mathcal{B}_{2 r}^{0}(z) \subset \dot{\mathcal{M}}$ for any $z \in \mathcal{D}$.

Let $B_{r}^{ \pm} \subset \mathbb{R}^{n_{ \pm}}$be the ball of center 0 and radius $r$, set $\mathcal{B}_{r}=B_{r}^{-} \times B_{r}^{+}$and let $X=\left(X_{0}, X_{-}, X_{+}\right) \in C^{2}\left(\mathcal{M} \times \mathcal{B}_{r}, \mathbb{R}^{n_{0}} \times \mathbb{R}^{n_{-}} \times \mathbb{R}^{n_{+}}\right)$. We consider the system: ${ }^{1}$

$$
\begin{aligned}
\dot{z} & =X_{0}(z, \chi) \\
\dot{\chi}_{-} & =\lambda(z) \chi_{-}+X_{+}(z, \chi) \\
\dot{\chi}_{+} & =-\lambda(z) \chi_{+}+X_{+}(z, \chi)
\end{aligned}
$$

where $\chi=\left(\chi_{-}, \chi_{+}\right) \in \mathcal{B}^{r}, z \in \mathcal{M}$. We assume:

$$
X=O_{2}(\chi), \min _{z \in \mathcal{M}} \lambda(z)=\delta>0 .
$$

Then we can perform the time change $d \tau=\lambda(z) d t$ and transform system (2.1) to the system

$$
\begin{aligned}
z^{\prime} & =Z_{0}(z, \chi) \\
\chi_{-}^{\prime} & =\chi_{-}+Z_{+}(z, \chi) \\
\chi_{+}^{\prime} & =-\chi_{+}+Z_{+}(z, \chi)
\end{aligned}
$$

where vector field $Z$ has the same properties of $X$. From now we will refer to system $(2.3)$.

Let $z_{0} \in \mathcal{D}$. The local stable and unstable manifolds $W_{l o c}^{s, u}\left(z_{0}\right)$ of system are represented by $C^{2}$ functions $g^{s}, f^{s}$ :

$$
\begin{aligned}
W_{l o c}^{s, u}\left(z_{0}\right) & =\left\{\left(z, \chi_{-}, \chi_{+}\right): z=g^{s, u}\left(z_{0}, \chi_{ \pm}\right), \chi_{\mp}\right. \\
& \left.=f^{s, u}\left(z_{0}, \chi_{ \pm}\right), \chi_{ \pm} \in \mathcal{B}_{\frac{r}{2}}^{ \pm}\right\} \\
f^{s, u}\left(z_{0}, \chi_{ \pm}\right) & =O_{2}\left(\chi_{ \pm}\right), \quad g^{s, u}\left(z_{0}, \chi_{ \pm}\right)=z_{0}+O_{2}\left(\chi_{ \pm}\right) .
\end{aligned}
$$

For any $\hat{\chi}_{+} \in B_{\frac{r}{2}}^{+}$let

$$
\begin{aligned}
\gamma^{s} & :[0, \infty) \rightarrow W_{l o c}^{s}\left(z_{0}\right), \\
\gamma^{s}(\tau) & =\phi_{s}^{\tau}\left(z_{0}, \hat{\chi}_{+}\right)=\phi^{\tau}\left(g^{s}\left(z_{0}, \gamma_{+}^{s}(\tau)\right), f^{s}\left(z_{0}, \gamma_{+}^{s}(\tau)\right), \gamma_{+}^{s}(\tau)\right)
\end{aligned}
$$

be the solution of $(2.1)$ on the stable manifold such that $\gamma_{+}^{s}(0)=\hat{\chi}_{+}$.

\footnotetext{
${ }^{1}$ This system is highly nongeneric, however it appears in our main applications to Celestial Mechanics (see [2], [3]).
} 
Similarly for $\hat{\chi}_{-} \in \mathcal{B}_{\frac{r}{2}}^{-}$let

$$
\begin{aligned}
\gamma^{u} & :(-\infty, 0] \rightarrow W_{l o c}^{u}\left(z_{0}\right) \\
\gamma^{u}(\tau) & =\phi_{u}^{\tau}\left(z_{0}, \hat{\chi}_{-}\right)=\phi^{\tau}\left(g^{u}\left(z_{0}, \gamma_{-}^{u}(\tau)\right), \gamma_{-}^{u}(\tau), f^{u}\left(z_{0}, \gamma_{-}^{u}(\tau)\right)\right.
\end{aligned}
$$

be the solution of $(2.1)$ on the unstable manifold such that $\gamma_{-}^{u}(0)=\hat{\chi}_{-}$. We recall that $\gamma^{s}, \gamma^{u}$ satisfy:

$$
\begin{aligned}
& \gamma_{+}^{s}(\tau)=e^{-t} \hat{\chi}_{+}+\int_{0}^{\tau} e^{-\tau+\theta} Z_{+}\left(\gamma^{s}(\theta)\right) d \theta \\
& \gamma_{-}^{s}(\tau)=-\int_{\tau}^{\infty} e^{\tau-\theta} Z_{-}\left(\gamma^{s}(\theta)\right) d \theta \\
& \gamma_{0}^{s}(\tau)=z_{0}-\int_{\tau}^{\infty} Z_{0}\left(\gamma^{s}(\theta)\right) d \theta \\
& \gamma_{+}^{u}(\tau)=\int_{-\infty}^{\tau} e^{-\tau+\theta} Z_{+}\left(\gamma^{u}(\theta)\right) d \theta \\
& \gamma_{-}^{u}(\tau)=e^{\tau} \hat{\chi}_{-}+\int_{0}^{\tau} e^{\tau-\theta} Z_{-}\left(\gamma^{u}(\theta)\right) d \theta \\
& \gamma_{0}^{u}(\tau)=z_{0}+\int_{-\infty}^{\tau} Z_{0}\left(\gamma^{u}(\theta)\right) d \theta
\end{aligned}
$$

and the estimates:

$$
\begin{aligned}
& \left|\gamma_{+}^{s}(\tau)\right| \leq C\left|\hat{\chi}_{+}\right| e^{-\tau},\left|\gamma_{-}^{s}(\tau)\right| \leq C r^{2} e^{-2 \tau},\left|\gamma_{0}^{s}(\tau)-z_{0}\right| \leq C r^{2} e^{-2 \tau}, \tau \geq 0 \\
& \left|\gamma_{+}^{u}(\tau)\right| \leq C r^{2} e^{2 \tau},\left|\gamma_{-}^{u}(\tau)\right| \leq C\left|\hat{\chi}_{-}\right| e^{\tau},\left|\gamma_{0}^{u}(\tau)-z_{0}\right| \leq C r^{2} e^{2 \tau}, \tau \leq 0
\end{aligned}
$$

where $C$ is a positive constant. ${ }^{2}$

Now we state the main Theorem:

Theorem 2.1. There exists $r \in(0,1)$ such that for any $z_{0} \in \mathcal{D}$, for any couple $\hat{\chi}_{-}, \hat{\chi}_{+}$such that $\left|\hat{\chi}_{ \pm}\right|=\frac{r}{2}$ and any $T^{\prime} \geq 1$ :

- There exists a unique solution

$$
\gamma(\tau)=\left(\gamma_{0}(\tau), \gamma_{-}(\tau), \gamma_{+}(\tau)\right) \in \mathcal{M} \times \mathcal{B}_{r}, \quad \tau \in\left[-T^{\prime}, T^{\prime}\right]
$$

to system (2.3) such that

$$
\gamma_{0}(0)=z_{0}, \quad \gamma_{ \pm}\left(\mp T^{\prime}\right)=\hat{\chi}_{ \pm} .
$$

Furthermore $\gamma$ smoothly depends on $\left(z_{0}, \hat{\chi}_{-}, \hat{\chi}_{+}, T^{\prime}\right) \in \mathcal{D} \times \mathcal{B} \times$ $[1, \infty)$.

\footnotetext{
${ }^{2}$ From now onward $C$ is the biggest of all possible constants entering in the inequalities of this section.
}

São Paulo J.Math.Sci. 6, 2 (2012), 351-363 
- We have the following representation of $\gamma$ :

$\gamma(\tau)=\gamma^{s}\left(T^{\prime}+\tau\right)+\gamma^{u}\left(-T^{\prime}+\tau\right)+V\left(z_{0}, \hat{\chi}_{-}, \hat{\chi}_{+}, \tau, T^{\prime}\right)$,

where $\gamma_{+}^{s}(0)=\hat{\chi}_{+}, \gamma_{-}^{u}(0)=\hat{\chi}_{-}$and $V$ is $C^{2}$ bounded on $\mathcal{D} \times B_{r} \times$ $\times\left[-T^{\prime}, T^{\prime}\right]$. Moreover there exists a positive constant $C$ such that $V \leq C T^{\prime} e^{-T^{\prime}} r^{2}$. More precisely:

$$
V_{0} \leq C r^{2} T^{\prime} e^{-2 T^{\prime}}, V_{ \pm} \leq C r^{2} e^{-2 T^{\prime}}
$$

The solution $\gamma$ is said to be a connecting solution to system (2.3).

Remark 2.1. Of course, coming back to the original time $t$ we gain the same results for system(2.1). To be explicit, setting $\tilde{\gamma}(t)=\gamma(\tau(t))$ and analogous for $\tilde{\gamma}^{s, u}$, we get from (2.10) the result:

$\tilde{\gamma}(\tau)=\tilde{\gamma}^{s}(T+t)+\tilde{\gamma}^{u}(-T+t)-\left(z_{0}, 0,0\right)+\tilde{V}\left(z_{0}, \hat{\chi}_{-}, \hat{\chi}_{+}, t, T\right), t \in[-T, T]$,

$$
\begin{aligned}
T & =\int_{0}^{T^{\prime}} \frac{d \theta}{\lambda\left(\gamma_{0}(\theta)\right)}=\frac{T^{\prime}}{\lambda\left(z_{0}\right)}\left(1+O\left(r^{2}\right)\right), \tilde{V}_{0} \leq C r^{2} T e^{-2 \lambda\left(z_{0}\right) T}, \tilde{V}_{ \pm} \\
& \leq C r^{2} e^{-2 \lambda\left(z_{0}\right) T} .
\end{aligned}
$$

Proof. The proof of Theorem (2.1) is divided in two steps.

2.1. First step. Fix $T^{\prime} \geq 1$ and consider the boundary conditions

$$
\begin{aligned}
z(0) & =z_{0} \\
\chi_{-}\left(T^{\prime}\right) & =\hat{\chi}_{-} \\
\chi_{+}\left(-T^{\prime}\right) & =\hat{\chi}_{+} .
\end{aligned}
$$

The equivalent integral version of the problem is:

$$
\begin{aligned}
z(\tau) & =z_{0}+\int_{0}^{\tau} Z_{0}(z(s), \chi(s)) d s \\
\chi_{-}(\tau) & =e^{\tau}\left\{e^{-T^{\prime}} \hat{\chi}_{-}-\int_{\tau}^{T^{\prime}} e^{-s} Z_{-}(z(s), \chi(s)) d s\right\} \\
\chi_{+}(\tau) & =e^{-\tau}\left\{e^{-T^{\prime}} \hat{\chi}_{+}+\int_{-T^{\prime}}^{\tau} e^{s} Z_{+}(z(s), \chi(s)) d s\right\} .
\end{aligned}
$$

Consider the Banach space $\mathcal{X}$ :

$$
\begin{aligned}
\mathcal{X}=\left\{\phi:=\left(\phi_{0}, \phi_{-}, \phi_{+}\right) \in C^{0}\left(\left[-T^{\prime}, T^{\prime}\right], \mathbb{R}^{2 n}\right):\right. \\
\left.\left.\quad \max \left\{\max _{\tau \in\left[-T^{\prime}, T^{\prime}\right]}\left|\phi_{0}(\tau)\right|, \max _{\tau \in\left[-T^{\prime}, T^{\prime}\right]}\left|\phi_{ \pm}(\tau)\right|\right\}<\infty\right\}\right\}
\end{aligned}
$$


with the uniform norm

$$
\|\phi\|:=\max \left\{\max _{\tau \in\left[-T^{\prime}, T^{\prime}\right]}\left|\phi_{0}(\tau)\right|, \max _{\tau \in\left[-T^{\prime}, T^{\prime}\right]}\left|\phi_{ \pm}(\tau)\right|\right\} .
$$

Then consider the closed subset of $\mathcal{X}$ :

$$
\begin{aligned}
\mathcal{X}_{r} & =\{\phi \in \mathcal{X}: \\
& \text { (i) } \phi_{0}(\tau) \in \mathcal{M}, \quad\left|\phi_{ \pm}(\tau)\right| \leq 2 r e^{-T^{\prime}} \cosh \tau, \quad \forall \tau \in\left[-T^{\prime}, T^{\prime}\right], \\
& \text { (ii) } \left.\phi_{0}(0)=z_{0}, \phi_{ \pm}\left(\mp T^{\prime}\right)=\hat{\chi}_{ \pm}\right\} .
\end{aligned}
$$

Solution of system (2.15) is obtained as the fixed point in $\mathcal{X}_{r}$ of the map $\mathcal{T}: \phi \rightarrow \tilde{\phi}$, acting on $\mathcal{X}_{r}$ and defined by:

$$
\begin{aligned}
& \tilde{\phi}_{0}(\tau)=z_{0}+\int_{0}^{\tau} Z_{0}(\phi(s)) d s \\
& \tilde{\phi}_{-}(\tau)=e^{\tau}\left\{e^{-T^{\prime}} \hat{\chi}_{-}-\int_{\tau}^{T^{\prime}} e^{-s} Z_{-}(\phi(s)) d s\right\} \\
& \tilde{\phi}_{+}(\tau)=e^{-\tau}\left\{e^{-T^{\prime}} \hat{\chi}_{+}+\int_{-T^{\prime}}^{\tau} e^{s} Z_{+}(\phi(s)) d s\right\} .
\end{aligned}
$$

First of all we prove

$$
\mathcal{T}: \mathcal{X}_{r} \rightarrow \mathcal{X}_{r}
$$

We recall:

$$
\max _{z \in \mathcal{M}}\left\{|Z(z, \chi)|,\left|D_{z} Z(z, \chi)\right|\right\} \leq C|\chi|^{2}, \max _{z \in \mathcal{M}}\left\{\left|D_{\chi_{ \pm}} Z(z, \chi)\right|\right\} \leq C|\chi|
$$

where $C$ is a positive real constant. Therefore for $|\tau| \leq T^{\prime}$ we have :

$$
\begin{gathered}
\int_{\tau}^{T^{\prime}} e^{\tau-s}\left|Z_{-}(\phi(s))\right| d s \leq C r^{2} e^{-T^{\prime}} \cosh \tau \\
\int_{-T^{\prime}}^{\tau} e^{-\tau+s}\left|Z_{+}(\phi(s))\right| d s \leq C r^{2} e^{-T^{\prime}} \cosh \tau
\end{gathered}
$$

and

$$
\left|\tilde{\phi}_{ \pm}(\tau)\right| \leq 2 e^{-T^{\prime}} \cosh \tau\left\{\left|\hat{\chi}_{ \pm}\right|+C r^{2}\right\} \leq r(1+2 C r) e^{-T^{\prime}} \cosh \tau .
$$

Moreover

$$
\left|\int_{0}^{|\tau|} Z_{0}(\phi(s)) d s\right| \leq \frac{C}{2} r^{2} e^{-2 T}(\sinh 2|\tau|+|\tau|) .
$$

Finally thanks to (2.22) and (2.23) considered for $r$ small enough, recalling also that $\forall z_{0} \in \mathcal{D}$ we have $B_{r}\left(z_{0}\right) \Subset \mathcal{M}$ we get

$$
\mathcal{T}: \mathcal{X}_{r} \rightarrow \mathcal{X}_{r}
$$


Consider now $\phi=\left(\phi_{0}, \phi_{-}, \phi_{+}\right)$and $\psi=\left(\psi_{0}, \psi_{-}, \psi_{+}\right)$belonging to $\mathcal{X}_{r}$ and their images through $\mathcal{T}, \tilde{\phi}=\left(\tilde{\phi}_{0}, \tilde{\phi}_{-}, \tilde{\phi}_{+}\right), \tilde{\psi}=\left(\tilde{\psi}_{0}, \tilde{\psi}_{-}, \tilde{\psi}_{+}\right)$. Taking into account (2.20) we have:

$$
\begin{aligned}
& |\tilde{\phi}(\tau)-\tilde{\psi}(\tau)| \\
\leq & C r\left\{e^{\tau} \int_{\tau}^{T^{\prime}} e^{-s}\left[\left|\phi_{-}(s)-\psi_{-}(s)\right|+\left|\phi_{+}(s)-\psi_{+}(s)\right|\right] d s\right. \\
+ & \left.e^{-\tau} \int_{-T^{\prime}}^{\tau} e^{s}\left[\left|\phi_{-}(s)-\psi_{-}(s)\right|+\left|\phi_{+}(s)-\psi_{+}(s)\right|\right] d s\right\} \\
+ & C r e^{-T^{\prime}} \int_{0}^{|\tau|} \cosh s\left|\phi_{0}(s)-\psi_{0}(s)\right| d s \\
\leq & 2 C r\|\phi-\psi\| .
\end{aligned}
$$

Therefore if $r$ is small enough the map $\mathcal{T}$ is a contraction and we get in $\mathcal{X}_{r}$ the fixed point $\gamma(\cdot):=\phi^{*}(\cdot \mid a):=\left(\gamma_{0}(\cdot \mid a), \phi_{-}^{*}(\cdot \mid a), \gamma_{+}(\cdot \mid a)\right)$, where

$$
a=\left(z_{0}, \hat{\chi}_{-}, \hat{\chi}_{+}, T^{\prime}\right) .
$$

The proof for continuous and differentiable dependence on $a$ follows by taking into consideration the variational equations of (2.15) (see [3]).

2.2. Second step. Let us introduce the following function $\Delta \in C^{1}\left(\left[-T^{\prime}, T^{\prime}\right], C^{2}\left(\mathcal{M} \times \mathcal{B}_{r}\right)\right)$ :

$$
\Delta(\tau)=\gamma(\tau)-\gamma^{s}\left(\tau+T^{\prime}\right)-\gamma^{u}\left(\tau-T^{\prime}\right)+\left(z_{0}, 0,0\right) .
$$

We take the following norm for $\Delta(\tau)$ :

$$
\begin{aligned}
|\Delta(\tau)|=\mid \gamma_{+} & (\tau)-\gamma_{+}^{s}\left(\tau+T^{\prime}\right)-\gamma_{+}^{u}\left(\tau-T^{\prime}\right) \mid \\
+ & \left|\gamma_{-}^{*}(\tau)-\gamma_{-}^{s}\left(\tau+T^{\prime}\right)-\gamma_{-}^{u}\left(\tau-T^{\prime}\right)\right| \\
& +\left|\gamma_{0}(\tau)-\gamma_{0}^{s}\left(\tau+T^{\prime}\right)-\gamma_{0}^{u}\left(\tau-T^{\prime}\right)+z_{0}\right| .
\end{aligned}
$$

We recall that from $(2.17),(2.23)$, we have

$$
\begin{aligned}
\left|\gamma_{ \pm}(\tau)\right| & \leq 2 r e^{|\tau|-T^{\prime}} \\
\left|\gamma_{0}(\tau)-z_{0}\right| & \leq 2 C r^{2} e^{2(|\tau|-T)}, \quad \tau \in\left[-T^{\prime}, T^{\prime}\right] .
\end{aligned}
$$

Moreover, considering $\gamma_{\lambda}(\theta)$ :

$$
\gamma_{\lambda}(\theta)=\lambda \gamma(\theta)+(1-\lambda) \gamma^{s}\left(\theta+T^{\prime}\right), \lambda \in[0,1],
$$

from (2.20) we have:

$$
\mid \frac{\partial Z_{\alpha}}{\partial z}\left(\gamma _ { \lambda } ( \theta ) | \leq C r ^ { 2 } e ^ { - 2 T ^ { \prime } + 2 | \theta | } , \quad | \frac { \partial Z _ { \alpha } } { \partial \chi _ { \pm } } \left(\gamma_{\lambda}(\theta) \mid \leq C r e^{-T+|\theta|} .\right.\right.
$$


Therefore if $\alpha \in\{0,-,+\}$ we have:

$$
\begin{gathered}
\left.\mid Z_{\alpha}(\gamma(\theta))-Z_{\alpha}\left(\gamma^{s}\left(\theta+T^{\prime}\right)\right)\right]\left|\leq C r e^{\left.|\theta|-T^{\prime}\right)}\left\{\mid \gamma_{+}(\theta)-\gamma_{+}^{s}\left(\theta+T^{\prime}\right)\right)\right| \\
\left.\left.\left.\quad+\mid \gamma_{-}^{*}(\theta)-\gamma_{-}^{s}\left(\theta+T^{\prime}\right)\right)|+| \gamma_{0}(\theta)-\gamma_{0}^{s}\left(\theta+T^{\prime}\right)\right) \mid r e^{\left.|\theta|-T^{\prime}\right)}\right\} \\
\left.\mid Z_{\alpha}(\gamma(\theta))-Z_{\alpha}\left(\gamma^{u}\left(\theta-T^{\prime}\right)\right)\right] \mid \leq C r\left[e^{|\theta|-T^{\prime}}\left\{\mid \gamma_{+}(\theta)-\gamma_{+}^{u}\left(\theta-T^{\prime}\right)\right) \mid\right. \\
\left.\left.\left.\quad+\mid \gamma_{-}^{*}(\theta)-\gamma_{-}^{u}\left(\theta-T^{\prime}\right)\right)|+| \gamma_{0}(\theta)-\gamma_{0}^{u}\left(\theta-T^{\prime}\right)\right) \mid r e^{\left.|\theta|-T^{\prime}\right)}\right\} .
\end{gathered}
$$

Consider $\tau \in\left[-T^{\prime}, 0\right]$ and set:

$$
\begin{aligned}
\Delta_{0}(\tau) & =\int_{0}^{\tau}\left[Z_{0}(\gamma(\theta))+\int_{-\infty}^{\tau} Z_{0}\left(\gamma^{u}\left(\theta-T^{\prime}\right)\right)+\int_{\tau}^{\infty} Z_{0}\left(\gamma^{s}\left(\theta+T^{\prime}\right)\right)\right] d \theta \\
\Delta_{-}(\tau) & =-\int_{\tau}^{T^{\prime}} e^{\tau-\theta}\left[Z_{-}(\gamma(\theta))-Z_{-}\left(\gamma^{s}\left(\theta+T^{\prime}\right)\right)-Z_{-}\left(\gamma^{u}\left(\theta-T^{\prime}\right)\right)\right] d \theta \\
& +\int_{T^{\prime}}^{\infty} e^{\tau-\theta} Z_{-}\left(\gamma^{s}\left(\theta+T^{\prime}\right)\right) d \theta \\
\Delta_{+}(\tau) & =-\int_{-T^{\prime}}^{\tau} e^{-\tau+\theta}\left[Z_{+}(\gamma(\theta))-Z_{+}\left(\gamma^{s}\left(\theta+T^{\prime}\right)\right)-Z_{+}\left(\gamma^{u}\left(\theta-T^{\prime}\right)\right)\right] d \theta \\
& +\int_{-\infty}^{-T^{\prime}} e^{-\tau+\theta} Z_{+}\left(\gamma^{u}\left(\theta-T^{\prime}\right)\right) d \theta
\end{aligned}
$$

We start with the evaluation of $\Delta_{0}(\tau)$. We have:

$$
\begin{aligned}
\left|\Delta_{0}(\tau)\right| \leq & \int_{\tau}^{0}\left|Z_{0}(\gamma(\theta))-Z_{0}\left(\gamma^{s}\left(\theta+T^{\prime}\right)\right)\right| d \theta+\int_{0}^{\infty} \mid Z_{0}\left(\gamma^{s}\left(\theta+T^{\prime}\right) \mid d \theta\right. \\
& +\int_{-\infty}^{\tau}\left|Z_{0}\left(\gamma^{u}\left(\theta-T^{\prime}\right)\right)\right| d \theta \\
\leq & C r \int_{\tau}^{0}\left\{e^{-(\theta+T)}\left\{\left|\gamma_{+}(\theta)-\gamma_{+}^{s}\left(\theta+T^{\prime}\right)\right|+\left|\gamma_{-}(\theta)-\gamma_{-}^{s}\left(\theta+T^{\prime}\right)\right|\right\} d \theta\right. \\
& \quad+C r^{2} e^{-2 T^{\prime}} \\
\leq & C r \int_{\tau}^{0} e^{-(\theta+T)}\left\{|\Delta(\theta)|+\left|\gamma_{0}^{u}\left(\theta-T^{\prime}\right)-z_{0}\right|\right] r e^{-(\theta+T)} \\
& \left.\quad+\left|\gamma_{+}^{u}\left(\theta-T^{\prime}\right)\right|+\gamma_{-}^{u}\left(\theta-T^{\prime}\right) \mid\right\} d \theta+C r^{2} e^{-2 T^{\prime}} . \quad(2.31)
\end{aligned}
$$

Taking into account the properties of $\gamma^{u}$ we get the conclusion:

$$
\left|\Delta_{0}(\tau)\right| \leq C r^{2}\left\{\int_{\tau}^{0} e^{-(\theta+T)}|\Delta(\theta)| d \theta+(|\tau|+1) e^{-2 T^{\prime}}\right\} .
$$


Consider now $\Delta_{-}(\tau)$. We have

$$
\begin{gathered}
\left|\Delta_{-}(\tau)\right| \leq \int_{\tau}^{T^{\prime}} e^{\tau-\theta}\left[Z_{-}(\gamma(\theta))-Z_{-}\left(\gamma^{s}\left(\theta+T^{\prime}\right)\right)-Z_{-}\left(\gamma^{u}\left(\theta-T^{\prime}\right)\right) \mid d \theta\right. \\
+C r^{2} e^{-4 T^{\prime}} \leq I_{1}+I_{2}+C r^{2} e^{-2 T^{\prime}}
\end{gathered}
$$

where

$$
\begin{aligned}
& I_{1}=\int_{\tau}^{0} e^{\tau-\theta}\left|Z_{-}(\gamma(\theta))-Z_{-}\left(\gamma^{s}\left(\theta+T^{\prime}\right)\right)\right| d \theta \\
& I_{2}=\int_{0}^{T^{\prime}} e^{\tau-\theta}\left|Z_{-}(\gamma(\theta))-Z_{-}\left(\gamma^{u}\left(\theta-T^{\prime}\right)\right)\right| d \theta .
\end{aligned}
$$

Let us show how we can estimate $I_{1}$. We have:

$$
\begin{aligned}
I_{1} & \leq C r \int_{\tau}^{0} e^{\tau-2 \theta-T^{\prime}}\left\{\left|\gamma_{+}(\theta)-\gamma_{+}^{s}\left(\theta+T^{\prime}\right)\right|\right. \\
& \left.\left.+\mid \gamma_{-}^{*}(\theta)-\gamma_{-}^{s}\left(\theta+T^{\prime}\right)\right)|+| \gamma_{0}(\theta)-\gamma_{0}^{s}\left(\theta+T^{\prime}\right) \mid r e^{-(\theta+T)}\right\} d \theta \\
& \leq C r \int_{\tau}^{0} e^{\tau-2 \theta-T^{\prime}}\left\{|\Delta(\theta)|+\mid \gamma_{+}^{u}\left(\theta-T^{\prime}\right)\right)\left|+\gamma_{-}^{u}\left(\theta-T^{\prime}\right)\right| \\
& \left.\left.+r e^{\theta-T^{\prime}} \mid \gamma_{0}^{s}\left(\theta+T^{\prime}\right)\right)-z_{0} \mid\right\} d \theta .
\end{aligned}
$$

Again taking into account the properties of $\gamma^{s, u}$, we get the conclusion:

$$
I_{1} \leq C r \int_{\tau}^{0} e^{\tau-2 \theta-T^{\prime}}|\Delta(\theta)| d \theta+C r^{2} e^{-2 T^{\prime}} .
$$

In analogous way we obtain the estimate for $I_{2}$

$$
I_{2} \leq C r e^{\tau-T^{\prime}} \int_{0}^{T^{\prime}}|\Delta(\theta)| d \theta+C r^{2} e^{-2 T^{\prime}}
$$

Finally we obtain

$$
I_{1} \leq C r \int_{\tau}^{0} e^{\tau-2 \theta-T^{\prime}}|\Delta(\theta)|+C r^{2} e^{-2 T^{\prime}}
$$

In the same way we obtain the estimate for $I_{2}$ :

$$
I_{2} \leq C r e^{\tau-T^{\prime}} \int_{0}^{T^{\prime}}|\Delta(\theta)|+C r^{2} e^{-2 T^{\prime}} .
$$

In conclusion we get the result:

$$
\begin{aligned}
\left|\Delta_{-}(\tau)\right| & \leq C r e^{\tau-T^{\prime}}\left\{\left|\int_{\tau}^{0} e^{-2 \theta}\right| \Delta(\theta)\left|+\int_{0}^{T^{\prime}}\right| \Delta(\theta) \mid\right\} \\
& +C r^{2} e^{-2 T^{\prime}}, \tau \in\left[-T^{\prime}, 0\right] .
\end{aligned}
$$


Finally we go to $\Delta_{+}(\tau)$. We have:

$$
\left|\Delta_{+}(\tau)\right| \leq \int_{-T^{\prime}}^{\tau} e^{-\tau+\theta}\left|Z_{+}\left(\phi^{*}(\theta)\right)-Z_{+}\left(\phi^{s}\left(\theta+T^{\prime}\right)\right)\right| d \theta+C r^{2} e^{-2 T^{\prime}}
$$

and in the same manner as before we gain the result:

$$
\left|\Delta_{+}(\tau)\right| \leq C r \int_{-T^{\prime}}^{\tau} e^{-\left(\tau+T^{\prime}\right)}|\Delta(\theta)|+C r^{2} e^{-2 T^{\prime}}, \tau \in\left[-T^{\prime}, 0\right] .
$$

For $\tau \in\left[0, T^{\prime}\right]$ similar inequalities are obtained interchanging the roles of $\phi^{s}\left(\theta+T^{\prime}\right)$ and $\phi^{u}\left(\theta-T^{\prime}\right)$ in (2.30). Finally, denoting by $M=\max _{\tau \in\left[-T^{\prime}, T^{\prime}\right]}|\Delta(\tau)|$, we obtain:

$$
\begin{aligned}
& \left|\Delta_{0}(\tau)\right| \leq C r^{2} M\left(T^{\prime} e^{-T^{\prime}}\right)+C r^{2} T^{\prime} e^{-2 T^{\prime}} \\
& \left|\Delta_{ \pm}(\tau)\right| \leq C r M+C r^{2} e^{-2 T^{\prime}}
\end{aligned}
$$

for any $\tau \in\left[-T^{\prime}, T^{\prime}\right]$. Then for $r$ small enough we get the result:

$$
\begin{aligned}
& \left|\Delta_{0}(\tau)\right| \leq C r^{2} T^{\prime} e^{-2 T^{\prime}} \\
& \left|\Delta_{ \pm}(\tau)\right| \leq C r^{2} e^{-2 T^{\prime}} .
\end{aligned}
$$

\section{The application to the three body problem}

In this section we show how results Section (2) can be applied to the $H_{\mu}$ hamiltonian system.

We perform the symplectic change of variables $\left(q_{1}, p_{1}, q_{2}, p_{2}\right) \rightarrow(x, y, u, v)$ :

$$
\begin{aligned}
& x=\alpha_{1} q_{1}+\alpha_{2} q_{2}, y=p_{1}+p_{2}, \quad u=q_{2}-q_{1} \\
& v=\alpha_{1} p_{2}-\alpha_{2} p_{1} .
\end{aligned}
$$

The variables $(u, v, z)$ are a version of Jacobi's variables. In the new variables the hamiltonian takes the form

$$
H_{\mu}=\frac{(1+\mu)|y|^{2}}{2}+\frac{|v|^{2}}{2 \alpha}-\frac{\alpha_{1}}{\left|\alpha_{2} u-x\right|}-\frac{\alpha_{2}}{\left|\alpha_{1} u+x\right|}-\frac{\mu \alpha}{|u|},
$$

where we denoted $\alpha=\alpha_{1} \alpha_{2}$. Consider negative energy $E$.

Collisions of $m_{1}, m_{2}$ with nonzero relative velocity correspond to the region $\mathcal{M}_{E}$ where

$$
K(z)=\frac{|y|^{2}}{2}-\frac{1}{|x|}<E
$$

Physically, $K$ is the Hamiltonian of motion of the center of mass of the bodies $m_{1}, m_{2}$ when they are glued together, forming a single body of mass $\alpha_{1}+\alpha_{2}=1$ which moves according to Kepler laws. The bodies $m_{1}, m_{2}$ 
collide if $K<E$ (if this is not the case, relative motion of planets is impossible). We introduce the function $\lambda(z)=E-K(z)$, define a function $\delta$ of $E$ (for instance $\delta(E)=\frac{1}{2}\left[\sqrt{E^{2}+2}+E\right]$ ) and consider the compact region $\mathcal{D}_{E} \Subset \mathcal{M}_{E}$ :

$$
\mathcal{D}_{E}=\left\{z \in \mathcal{M}_{E}: \lambda(z)>\delta \quad|x| \geq \delta\right\} .
$$

Then we perform the Levi-Civita regularization on the fixed energy level $\Sigma_{E}=\{H=E\}$, identifying $u, v \in \mathbb{R}^{2}=\mathbb{C}$ with complex numbers and making the symplectic change of variables

$$
g(\xi, \eta, z)=(u, v, z), \quad u=\xi^{2}, \quad v=\frac{\bar{\eta}}{2 \xi}, \quad \xi, \eta \in \mathbb{C} .
$$

In the new variables $(\xi, \eta, z)$ the hamiltonian $H_{\mu}$ becomes

$$
H_{\mu}=\frac{(1+\mu)|y|^{2}}{2}+\frac{|\eta|^{2}}{8 \alpha|\xi|^{2}}-\frac{\alpha_{1}}{\left|\alpha_{2} \xi^{2}-x\right|}-\frac{\alpha_{2}}{\left|\alpha_{1} \xi^{2}+x\right|}-\frac{\mu \alpha}{|\xi|^{2}}
$$

Orbits of system $\left(H_{\mu}\right)$ with energy $E$ are transformed into orbits of system $\left(\hat{H}_{\mu}\right)$, whose regularized hamiltonian is

$$
\hat{H}_{\mu}=\frac{|\eta|^{2}}{8 \alpha^{2}}-\frac{|\xi|^{2}}{\alpha}\left(E+\frac{\alpha_{1}}{\left|\alpha_{2} \xi^{2}-x\right|}+\frac{\alpha_{2}}{\left|\alpha_{1} \xi^{2}+x\right|}-\frac{(1+\mu)|y|^{2}}{2}\right)
$$

and $\mu$ is the new energy. To eliminate this dependence, one solves the equation $\hat{H}_{\mu}=\mu$ for $\mu=\mathcal{H}(\xi, \eta, z)$ in a tubular neighborhood $U$ of $\mathcal{D}_{E}$ obtaining $\mathcal{H}$ :

$$
\mathcal{H}(\xi, \eta . z)=\left(\frac{|\eta|^{2}}{8 \alpha^{2}}-\frac{|\xi|^{2}}{\alpha}\left(E+\frac{\alpha_{1}}{\left|\alpha_{2} \xi^{2}-x\right|}+\frac{\alpha_{2}}{\left|\alpha_{1} \xi^{2}+x\right|}-\frac{|y|^{2}}{2}\right)\right)\left(1-\frac{|\xi|^{2}|y|^{2}}{2 \alpha}\right)^{-1} .
$$

The level sets of $\mathcal{H}$ and $\hat{H}_{\mu}$ are the same in $U$ :

$$
\hat{\Sigma}_{\mu} \cap U=\left\{\hat{H}_{\mu}=\mu\right\} \cap U=\{\mathcal{H}=\mu\} \cap U
$$

and the orbits of system $\left(H_{\mu}\right)$ with energy $E$ which are near collision of $m_{1}, m_{2}$ are transformed into orbits of system $\mathcal{H}$ with energy $\mu$. Introduce the new variables $\chi=\left(\chi_{-}, \chi_{+}\right)$:

$$
\begin{aligned}
& \chi_{+}=2\left(\xi_{1} \sqrt{2 \lambda(z) \alpha}-\eta_{1}, \xi_{2} \sqrt{2 \lambda(z) \alpha}-\eta_{2}\right) \\
& \chi_{-}=2\left(\xi_{1} \sqrt{2 \lambda(z) \alpha}+\eta_{1}, \xi_{2} \sqrt{2 \lambda(z) \alpha}+\eta_{2}\right), z \in \mathcal{D}_{E} .
\end{aligned}
$$


After a suitable rescaling of time the hamiltonian system reduces to the system (2.1) having the energy first integral of the form:

$$
\begin{aligned}
\mathcal{H} & =-\left\langle\chi_{+}, \chi_{-}\right\rangle+Q\left(z, \chi_{-}, \chi_{+}\right) \\
Q\left(z, \chi_{-}, \chi_{+}\right) & =O_{2}\left(\chi_{-}, \chi_{+}\right) .
\end{aligned}
$$

We have to find connecting solution lying on the energy level set $\mu$ therefore we select time $T^{\prime}$ so that the corresponding connecting solution $\gamma$ in (2.10) lies on the level set $\mu$. To this purpose we consider the equation:

$$
\left.\mathcal{H}\right|_{\gamma}=\mu
$$

that is

$$
e^{-2 T^{\prime}}\left\{\left\langle\hat{\chi}_{+}, \hat{\chi}_{-}\right\rangle+O_{3}(\hat{\chi})+e^{-T^{\prime}} R\left(\hat{\chi}_{-}, \hat{\chi}_{+}, T^{\prime}\right)\right\}=\mu .
$$

(with $R\left(\hat{\chi}_{-}, \hat{\chi}_{+}, T^{\prime}\right)=O_{2}(\hat{\chi})$, uniformly with respect to $\left.T^{\prime}\right)$.

We set

$$
\begin{gathered}
\hat{\chi}_{+}=\frac{r}{2} e^{i \theta_{+}}, \hat{\chi}_{-}=\frac{r}{2} e^{i \theta_{-}}, \theta=\theta_{+}-\theta_{-}, \\
R\left(q_{+}, p_{-}, T^{\prime}\right)=r^{2} C\left(r, \theta_{+}, \theta_{-}, T^{\prime}\right),
\end{gathered}
$$

$G$ being a bounded function, and we write equation in the form

$$
r^{2} e^{-2 T^{\prime}}\left\{\cos \theta\left(\frac{1}{4}+e^{-T^{\prime}} G\left(r, \theta_{+}, \theta_{-}, T^{\prime}\right)+O_{1}(r)\right\}=\mu .\right.
$$

Assume $\cos \theta:=\epsilon>0$, write $\mu=k \epsilon r^{2}, k$ being a non negative parameter, so that (3.9) becomes:

$$
e^{-2 T^{\prime}}\left\{\frac{1}{4}+\frac{1}{\epsilon} e^{-T^{\prime}} C\left(r, \theta_{+}, \theta_{-}\right)+\frac{\left.O_{1}(r)\right)}{\epsilon}\right\}=k .
$$

The 1.h.s. of the equation is a monotone decreasing function of $T^{\prime}$, for $T^{\prime}>0$. Therefore there exists a function $T^{\prime}=T^{\prime}(k)$, which uniquely solves the equation in a right neighborhood of $k=0$. Moreover $\lim _{k \rightarrow 0^{+}} T^{\prime}(k)=$ $\infty$.

\section{References}

[1] Bolotin S., Second species periodic orbits of the elliptic 3 body problem. Celest. Mech. Dynam. Astron., 93 (2006), 345-373.

[2] Bolotin S., Negrini P., Variational approach to second species periodic solutions of Poincaré of the 3 body problem. Discr. Conts. Dynam. Syst., 33 (March 2013), no.3,1009-2013

[3] Bolotin S. and Negrini P., Shilnikov Lemma for a nondegenerate critical manifold of a Hamiltonian system. In preparation.

[4] Bolotin S. V. and MacKay R. S., Periodic and chaotic trajectories of the second species for the $n$-centre problem, Celest. Mech. and Dynam. Astron., 77 (2000), 49-75.

[5] Marco J.-P. and Niederman L., Sur la construction des solutions de seconde espèce dans le problème plan restreint des trois corps, Ann. Inst. H. Poincare Phys. Théor., 62 (1995), 211-249. 
[6] Poincaré, A. Les Méthodes Nouvelles de la Mécanique Céleste, Volume 3.

[7] Turaev, D. V. and Shilnikov, L. P., Hamiltonian systems with homoclinic saddle curves. (Russian) Dokl. Akad. Nauk SSSR 304, no. 4 (1989), 811-814; (translation in Soviet Math. Dokl., 39, no. 1, (1989), 165-168). 